\title{
A Lieb-Thirring bound for a magnetic Pauli Hamiltonian, II
}

Luca Bugliaro, Charles Fefferman and Gian Michele Graf

\begin{abstract}
We establish a Lieb-Thirring type estimate for Pauli Hamiltonians with non-homogeneous magnetic fields. Besides of depending on the size of the field, the bound also takes into account the size of the field gradient. We then apply the inequality to prove stability of non-relativistic quantum mechanical matter coupled to the quantized ultraviolet-cutoff electromagnetic field for arbitrary values of the fine structure constant.
\end{abstract}

\section{Introduction.}

We continue here our analysis of Lieb-Thirring type estimates for Pauli Hamiltonians, which we begun in [1] (henceforth called I) and present its applications to the stability of matter coupled to the (ultraviolet-cutoff) quantized electromagnetic field. The one-particle Hamiltonian we consider describes a spin $1 / 2$ electron and is once more

$$
H=\not D^{2}-V
$$

acting on $\mathcal{H}=L^{2}\left(\mathbb{R}^{3}\right) \otimes \mathbb{C}^{2}$, where $D=p-A$ and $\not D=D \cdot \sigma$. Here, $A(x)$ is the magnetic vector potential, $\sigma$ is the vector of Pauli matrices, and $V(x) \geq 0$ is a scalar potential. In I, the paradigm was given by the well-known Lieb-Thirring estimate [11] for the case $B=\nabla \wedge A=0$ and our estimate (I.1.2) aimed at estimating the effect of $B \neq 0$ (see 
[4], [9], [17], [18], [5], [15] for other results in this direction). Here, by contrast, the starting point is the following bound, due to Lieb, Solovej and Yngvason [10], on the sum of the negative eigenvalues $-e_{i}$ of (1.1),

$$
\sum e_{i} \leq C \int V(x)^{3 / 2}(V(x)+B) d^{3} x
$$

which holds for the case in which the field $B$ is constant. Our goal is to generalize it to the case where $B$ is not constant, or, more precisely, that of estimating the effect of $\nabla \otimes B=\left(\partial_{i} B_{j}\right)_{i, j=1,2,3} \neq 0$ on (1.2). We remark that an estimate having the same purpose, but quite different assumptions on $B$, has been derived in [5], [6].

In $\mathrm{I}$, the role of $B(x)$ was expressed by means of a length scale $r(x)$ defined through $B(x)$ non-locally (incorporating insight of [4], [17], $[18])$. Similarly here, the role of $\nabla \otimes B$ will be reflected in a second length scale $l(x)$. These two length scales satisfy

$$
\begin{gathered}
\int r(x)^{-4} d^{3} x \leq C \int B(x)^{2} d^{3} x \\
\int l(x)^{-6} d^{3} x \leq C \int(\nabla \otimes B(x))^{2} d^{3} x
\end{gathered}
$$

as well as some local variants thereof. We can now state our generalization of (1.2).

Theorem 1. For sufficiently small $\varepsilon>0$ there are constants $C^{\prime}, C^{\prime \prime}>$ 0 such that for any vector potential $A \in L_{\mathrm{loc}}^{2}\left(\mathbb{R}^{3}, \mathbb{R}^{3}\right)$

$$
\begin{aligned}
\sum e_{i} \leq & C^{\prime} \int V(x)^{3 / 2}(V(x)+\widehat{B}(x)) d^{3} x \\
& +C^{\prime \prime} \int V(x) P(x)^{1 / 2}(P(x)+\widehat{B}(x)) d^{3} x
\end{aligned}
$$

where $\widehat{B}(x)$ is the average of $|B(y)|$ over a ball of radius $\varepsilon l(x)$ centered at $x$, and

$$
P(x)=l(x)^{-1}\left(r(x)^{-1}+l(x)^{-1}\right) .
$$

As noticed in [5], (1.5) yields, by the variational principle, a bound on the density $n(x)=E(x, x)$ of zero modes of $\not D$, where $E(x, y)$ is 
the integral kernel of the spectral projection $E$ corresponding to the possible [13] eigenvalue 0 of $\not D$. The bound is

$$
n(x) \leq C^{\prime \prime} P(x)^{1 / 2}(P(x)+\widehat{B}(x)),
$$

and, as it should, it vanishes in the case of a homogeneous magnetic field, where $l=\infty$.

In Section 2 we discuss the properties of the two length scales mentioned above. The main part of the proof of Theorem 1 is given in Section 3, while some more technical aspects are deferred to Section 4. In order to keep these sections reasonably short we shall be brief on details which have already been discussed at length in I.

We now turn to the implications of estimate (1.5) regarding stability of non-relativistic matter coupled to quantum electromagnetic field. We recover a result of [8] establishing stability for any value of the fine structure constant $\alpha$, with a bound depending however on the ultraviolet cutoff $\Lambda<\infty$. The details of the model are as follows. The electromagnetic vector potential is (in appropriate units [2])

$$
\begin{aligned}
& A_{\Lambda}(x) \equiv A(x)=A_{-}(x)+A_{+}(x), \quad A_{+}(x)=A_{-}(x)^{*}, \\
& A_{-}(x)=\frac{\alpha^{1 / 2}}{2 \pi} \int \kappa(k)|k|^{-1 / 2} \sum_{\lambda= \pm} a_{\lambda}(k) e_{\lambda}(k) e^{i k x} d^{3} k .
\end{aligned}
$$

The cutoff function $\kappa(k)$ satisfies $|\kappa(k)| \leq 1$ and $\operatorname{supp} \kappa \subset\left\{k \in \mathbb{R}^{3}\right.$ : $|k| \leq \Lambda\}$; the operators $a_{\lambda}(k)^{*}$ and $a_{\lambda}(k)$ are creation and annihilation operators on the bosonic Fock space $\mathcal{F}$ over $L^{2}\left(\mathbb{R}^{3}\right) \otimes \mathbb{C}^{2}$ (with $\mathbb{C}^{2}$ accounting for the helicity states of the photon) and satisfy canonical commutation relations

$$
\left[a_{\lambda}(k)^{\#}, a_{\lambda^{\prime}}\left(k^{\prime}\right)^{\#}\right]=0, \quad\left[a_{\lambda}(k), a_{\lambda^{\prime}}\left(k^{\prime}\right)^{*}\right]=\delta_{\lambda \lambda^{\prime}} \delta\left(k-k^{\prime}\right) .
$$

Moreover, for each $k$, the direction of propagation $\hat{k}=k /|k|$ and the polarizations $e_{ \pm}(k) \in \mathbb{C}^{3}$ are orthonormal. The free photon Hamiltonian is

$$
H_{f}=\alpha^{-1} \int|k| \sum_{\lambda= \pm} a_{\lambda}(k)^{*} a_{\lambda}(k) d^{3} k .
$$

Matter consists of $K$ nuclei of charge $Z>0$ with arbitrary positions $R_{k},(k=1, \ldots, K)$ and $N$ electrons obeying the Pauli principle. The Hamiltonian for both matter and field, acting on $\left(\wedge^{N} \mathcal{H}\right) \otimes \mathcal{F}$, is

$$
H=H_{m}+H_{f},
$$


where

$$
\begin{gathered}
H_{m}=\sum_{i=1}^{N} \not D_{i}^{2}+V_{C} \\
V_{C}=\sum_{\substack{i, j=1 \\
i<j}}^{N} \frac{1}{\left|x_{i}-x_{j}\right|}-\sum_{i, k=1}^{N, K} \frac{Z}{\left|x_{i}-R_{k}\right|}+\sum_{\substack{k, l=1 \\
k<l}}^{K} \frac{Z^{2}}{\left|R_{k}-R_{l}\right|} .
\end{gathered}
$$

The energy per particle is bounded below as shown by the following result, previously established in [8].

Theorem 2. The Hamiltonian $H$ satisfies

$$
H \geq-C(Z, \alpha, \Lambda)(N+K),
$$

where

$$
C(Z, \alpha, \Lambda)=\text { const } z^{* 5} \log \left(1+z^{*}\right) Z^{*}\left(\Lambda+z^{*-2} Z^{*}\right),
$$

with $z^{*}=1+Z^{*} \alpha^{2}$ and $Z^{*}=Z+1$.

The proof, given in Section 6, rests on a stability result [7] for matter coupled to a classical magnetic field, which is here established in Section 5. This is actually where estimate (1.5) enters.

\section{The basic length scales.}

We define the length scales we mentioned in the introduction as the solutions $r=r(x)>0$ respectively $l=l(x)>0$ of the equations

$$
\begin{gathered}
r \int \varphi\left(\frac{y-x}{r}\right) B(y)^{2} d^{3} y=1, \\
l^{3} \int \varphi\left(\frac{y-x}{l}\right)(\nabla \otimes B(y))^{2} d^{3} y=1 .
\end{gathered}
$$

The function $\varphi: \mathbb{R}^{3} \longrightarrow \mathbb{R}, \varphi(z)=\left(1+z^{2} / 2\right)^{-2}$ is the same as in I and satisfies

$$
\begin{gathered}
z \cdot \nabla \varphi(z) \leq 0, \\
\left|D_{1} \cdots D_{n} \varphi\right| \lesssim \varphi, \quad n \in \mathbb{N},
\end{gathered}
$$


where $D_{j}=\partial_{i},(i=1,2,3)$ or $D_{j}=z \cdot \nabla$. Here and in the following $X \lesssim Y$ means $X \leq C Y$ for some constant $C$ independent of the data, i.e., of $A, V$.

The solutions of (2.1) and (2.2) exist and are unique, except for the case $B \equiv 0$ (almost everywhere), respectively $\nabla \otimes B \equiv 0$ (almost everywhere), where we set $r \equiv \infty$, respectively $l \equiv \infty$. They are smooth as a function of $x \in \mathbb{R}^{3}$ (see Section I.2).

We first discuss how these length scales are semi-locally controlled by the original quantities $B$ and $\nabla \otimes B$. To this end let $\Omega_{R}=\{x$ : dist $(x, \Omega)<R\}$ for $R>0$ and $\Omega \subset \mathbb{R}^{3}$.

Lemma 3. The length scales $r(x)$ and $l(x)$ satisfy (1.3), (1.4). Moreover, for any $R>0$ and $\Omega \subset \mathbb{R}^{3}$ there is a function $\Phi_{\Omega, R}(x) \geq 0$ satisfying $\left\|\Phi_{\Omega, R}\right\|_{\infty} \lesssim 1$ and $\left\|\Phi_{\Omega, R}\right\|_{1} \lesssim\left|\Omega_{R}\right|$, uniformly in $\Omega, R$, such that

$$
\begin{gathered}
\int_{\Omega_{R}} r(x)^{-4} d^{3} x \lesssim \int \Phi_{\Omega, R}(x) B(x)^{2} d^{3} x+\left|\Omega_{R}\right| R^{-4} \\
\int_{\Omega_{R}} l(x)^{-6} d^{3} x \lesssim \int \Phi_{\Omega, R}(x)(\nabla \otimes B(x))^{2} d^{3} x+\left|\Omega_{R}\right| R^{-6}
\end{gathered}
$$

Proof. Estimates (1.3) and (2.5) were proven in Lemmas I.2 and I.12. The same proofs are valid for the remaining two estimates once the following remark about the proof of Lemma I.2 has been made: We replace there $r(x)$ by $l(x)$. Because of $g_{+}(|x|) \geq 1$, (I.2.6) implies

$$
g_{+}(|x|)^{3} \varphi\left(\frac{z-x}{g_{+}(|x|)}\right) \geq \varphi(z),
$$

which after integration against $(\nabla \otimes B(z))^{2} d^{3} z$ implies $l(x) \leq g_{+}(|x|)$. Then the proof continues as before.

The length scales $r(x)$ and $l(x)$ are tempered in the following sense:

\section{Lemma 4.}

$$
\begin{gathered}
\left|\partial^{\alpha} l(x)\right| \lesssim l(x)^{-(|\alpha|-1)}, \quad|\alpha| \geq 0 \\
\left|\partial^{\alpha} r(x)\right| \lesssim r(x)^{-(|\alpha|-1)} \min \left\{1,\left(\frac{r(x)}{l(x)}\right)^{3 / 2}\right\}, \quad|\alpha| \geq 1
\end{gathered}
$$


where $\alpha \in \mathbb{N}^{3}$ is a multiindex.

Proof. We omit the proof of (2.7) since it consists of a minor adaptation of that of (I.2.9). For $r(x)>l(x)(2.8)$ reduces to (I.2.9), so that we may assume $r(x)<l(x)$. We discuss this case using a variant of the argument given in I. We recall that it was based on the equation

$$
(1-m(x)) \partial_{i} r(x)=m_{i}(x),
$$

where

$m(x)=r(x) \int z \cdot \nabla \varphi(z) U(y) d^{3} y, \quad m_{i}(x)=r(x) \int\left(\partial_{i} \varphi\right)(z) U(y) d^{3} y$,

with $z=(y-x) / r(x)$. Moreover, we denoted by $V_{n}, n \in \mathbb{N}$, the space of finite sums of functions of the form

$$
f(x)=r(x)^{-(n-1)} P\left(\left\{\partial^{\alpha} r\right\}\right) \int \psi(z) B(y)^{2} d^{3} y,
$$

where $\psi$ is of the form $D_{1} \cdots D_{k} \varphi$ and $P$ is a monomial in the derivatives $\left\{\partial^{\alpha} r\right\}_{|\alpha| \leq n}$ of order 0 in the sense that it contains as many powers of $\partial$ as of $r$. In addition we consider here the subspace $\widetilde{V}_{n} \subset V_{n}$ obtained by restricting $f$ to satisfy: i) some $\partial^{\alpha} r$ with $1 \leq|\alpha| \leq n$ occurs among the factors of $P$; or else ii) $D_{1}=\partial_{i}$, i.e., $\psi=\partial_{i} \widetilde{\psi}$ with $\widetilde{\psi}$ of the form previously stated for $\psi$. One verifies that $\partial_{i} V_{n} \subset \widetilde{V}_{n+1}$ and $r^{-1} \widetilde{V}_{n} \subset$ $\widetilde{V}_{n+1}$.

The induction assumption states that (2.8) holds for $1 \leq|\alpha| \leq n$. (It is empty for $n=0$ ). We now prove it for $n+1$ instead of $n$. First, we claim that $f \in \widetilde{V}_{n}$ satisfies

$$
|f(x)| \lesssim r(x)^{-n}\left(\frac{r(x)}{l(x)}\right)^{3 / 2} .
$$

In case i) this follows directly from the induction assumption; in case ii) by integration by parts

$$
\int \partial_{i} \widetilde{\psi}(z) B(y)^{2} d^{3} y=2 r(x) \int \widetilde{\psi}(z) B(y) \cdot \partial_{i} B(y) d^{3} y,
$$

which by (2.4) and the Cauchy-Schwarz inequality is bounded in absolute value by

$$
\begin{aligned}
2 r(x)\left(\int \varphi(z) B(y)^{2} d^{3} y\right)^{1 / 2}\left(\int \varphi(z)(\nabla \otimes B(y))^{2} d^{3} y\right)^{1 / 2} & \\
& \lesssim r(x)^{-1}\left(\frac{r(x)}{l(x)}\right)^{3 / 2} .
\end{aligned}
$$


In the last estimate we used that the first integral equals $r(x)^{-1}$, whereas the second may be estimated by replacing $z$ by $(y-x) / l(x)$, since $r(x)^{-1}>l(x)^{-1}$ and $\varphi(z)$ is radially decreasing. Hence that integral is bounded by $l(x)^{-3}$. We can turn to (2.8): Applying $\partial^{\alpha},(|\alpha|=n)$ to (2.9) and using $m \in V_{0}$ we obtain $(1-m(x)) \partial^{\alpha} \partial_{i} r(x) \in \partial^{\alpha} m_{i}+\widetilde{V}_{n}$. The last set is $\widetilde{V}_{n}$ (even for $|\alpha|=n=0$ ), since $m_{i} \in \widetilde{V}_{0}$. The result follows with $m \leq 0$.

We remark that (2.7) implies (see (I.2.13))

$$
|x-y| \leq \varepsilon l(x) \quad \text { implies } \quad \frac{1}{2} \leq \frac{l(y)}{l(x)} \leq 2
$$

for $\varepsilon>0$ small enough. A partition of unity based on the length scale $l(x)$ is

$$
j_{y}(x)=(\varepsilon l(x))^{-3 / 2} \chi\left(\frac{x-y}{\varepsilon l(x)}\right), \quad y \in \mathbb{R}^{3},
$$

where $0<\varepsilon \leq 1$ and $\chi \in C_{0}^{\infty}\left(\mathbb{R}^{3}\right)$ with supp $\chi \subset\{z:|z| \leq 1\}$ and $\int \chi(z)^{2} d^{3} z=1$. Analogously to Lemma I.4 we have

\section{Lemma 5.}

$$
\begin{gathered}
\int j_{y}(x)^{2} d^{3} y=1 \\
\int\left|\partial^{\alpha} j_{y}(x) \partial^{\beta} j_{y}(x)\right| d^{3} y \lesssim(\varepsilon l(x))^{-(|\alpha|+|\beta|)},
\end{gathered}
$$

for any $\alpha, \beta \in \mathbb{N}^{3}$, where $\partial=\partial / \partial x$.

The length scale $l(x)$ will be the one most frequently used in the following sections. At one point however (in the proof of Lemma 8), we will use the length scale $\lambda(x)$ defined by $\lambda(x)^{-1}=r(x)^{-1}+l(x)^{-1}$. It also satisfies (2.7) and (2.10) (with $l$ replaced by $\lambda$ ), and Lemma 5 applies accordingly to the partition based on $\lambda(x)$.

Finally we point out that Lemma 4 (in particular, the improvement of (2.8) over (I.2.9)) implies

$$
|\nabla P(x)| \lesssim P(x) l(x)^{-1}, \quad|\Delta P(x)| \lesssim P(x)^{2} .
$$


Combining (2.13) with (2.10) we also find that for $|x-y| \leq \varepsilon l(x)$ we have $|\log P(y)-\log P(x)| \lesssim \varepsilon$, and hence

$$
\frac{1}{2} \leq \frac{P(y)}{P(x)} \leq 2
$$

for $\varepsilon>0$ small enough.

\section{The eigenvalue sum.}

In this section we present the framework of the proof of (1.5), with large parts of it deferred to the next section. We begin by applying, as in I, the Birman-Schwinger principle [14]

$$
\left.\sum e_{i} \leq 2 \int_{0}^{\infty} n\left((\not)^{2}+E\right)^{-1 / 2}(V-E)_{+}^{1 / 2}, 1\right) d E
$$

where $n(X, \mu)$ is the number of singular values $\lambda \geq \mu>0$ of a compact operator $X$, i.e., the number of eigenvalues $\lambda^{2} \geq \mu^{2}$ of $X^{*} X$. We then decompose the operator in $(3.1)$ as $K_{>}(E)+K_{<}(E)$ with

$$
\begin{gathered}
K_{>}(E)=\left(\not D^{2}+\varepsilon^{-3} P+E\right)^{-1 / 2}(V-E)_{+}^{1 / 2} \\
K_{<}(E)=\left(\left(\not D^{2}+E\right)^{-1 / 2}-\left(\not D^{2}+\varepsilon^{-3} P+E\right)^{-1 / 2}\right)(V-E)_{+}^{1 / 2}
\end{gathered}
$$

for some sufficiently small $\varepsilon>0$, and note that (see e.g. [3], [19])

$$
n\left(K_{>}+K_{<}, s_{1}+s_{2}\right) \leq n\left(K_{>}, s_{1}\right)+n\left(K_{<}, s_{2}\right),
$$

(we take $s_{1}=s_{2}=1 / 2$ ). For the last term we shall prove the bound

$$
n\left(K_{<}(E), \frac{1}{2}\right) \lesssim n\left(\left(\not D^{2}+\varepsilon^{-3} P\right)^{-1} \varepsilon^{-3} P V^{1 / 2}, \text { const } E^{1 / 2}\right) .
$$

For the purpose of estimating $n\left(K_{<}, 1 / 2\right)$ and $n\left(K_{>}, 1 / 2\right)$ we introduce some auxiliary objects, starting with the Hilbert space $\widehat{\mathcal{H}}=\int_{\mathbb{R}^{3}}^{\oplus} \mathcal{H} d^{3} y$ and the linear map

$$
J: \mathcal{H} \longrightarrow \widehat{\mathcal{H}}, \quad J=\int_{\mathbb{R}^{3}}^{\oplus} j_{y} d^{3} y,
$$


(see also Section I.3). Next we define

$$
\widehat{H}: \widehat{\mathcal{H}} \longrightarrow \widehat{\mathcal{H}}, \quad \widehat{H}=\int_{\mathbb{R}^{3}}^{\oplus} e^{i f_{y}} H_{y} e^{-i f_{y}} d^{3} y,
$$

where $H_{y}=H\left(B_{y}\right)+\varepsilon^{-3} P(y), H(B)=((p-(1 / 2) B \wedge x) \cdot \sigma)^{2}, f_{y}(x)$ is a function to be specified later and $B_{y}=\left|K_{y}\right|^{-1} \int_{K_{y}} B(x) d^{3} x$ is the average magnetic field in the ball $K_{y}=\{x:|x-y|<2 \varepsilon l(y)\}$. In summary, $\widehat{H}$ acts on fibers of $\widehat{\mathcal{H}}$ as a Pauli Hamiltonian with constant magnetic field. The Pauli operator $\not D^{2}$ compares to the above construction as

$$
\left(\not D^{2}+\varepsilon^{-3} P\right)^{2} \gtrsim J^{*} \widehat{H}^{2} J
$$

This inequality, which is at the center of our analysis, is obtained by first localizing $\left(\not D^{2}+\varepsilon^{-3} P\right)^{2}$ and then by locally replacing the fields $B=\nabla \wedge A$ by a constant magnetic field and $P$ by a constant. Indeed, (3.4) results from the combination of the following two inequalities.

\section{Lemma 6.}

$$
\begin{gathered}
\left(\not D^{2}+\varepsilon^{-3} P\right)^{2} \geq \int j_{y}\left(\not D^{4}+\frac{1}{2} \varepsilon^{-6} P^{2}\right) j_{y} d^{3} y \\
j_{y}\left(\not D^{4}+\frac{1}{2} \varepsilon^{-6} P^{2}\right) j_{y} \gtrsim j_{y} H_{y}^{2} j_{y} .
\end{gathered}
$$

Let us point out that (3.4) implies the weaker inequality (see

$$
\not D^{2}+\varepsilon^{-3} P \gtrsim J^{*} \widehat{H} J
$$

ProOF OF (1.5). Let

$$
\widehat{H}^{0}: \widehat{\mathcal{H}} \longrightarrow \widehat{\mathcal{H}}, \quad \widehat{H}^{0}=\int_{\mathbb{R}^{3}}^{\oplus} e^{i f_{y}} H\left(B_{y}\right) e^{-i f_{y}} d^{3} y .
$$

Then $\widehat{H} \geq \widehat{H}^{0}$ and, as in I, we obtain from (3.7)

$$
n\left(K_{>}(E), \frac{1}{2}\right) \leq n\left(\left(\widehat{H}^{0}+E\right)^{-1 / 2} J(V-E)_{+}^{1 / 2}, \text { const }\right)
$$


by means of (3.7). From now on the computation closely follows the line given in [10], where the contribution of the lowest Landau band is split from that of the higher bands. We set

$$
\widehat{\Pi}: \widehat{\mathcal{H}} \longrightarrow \widehat{\mathcal{H}}, \quad \widehat{\Pi}=\int_{\mathbb{R}^{3}}^{\oplus} e^{i f_{y}} \Pi\left(B_{y}\right) e^{-i f_{y}} d^{3} y
$$

where $\Pi(B)$ is the projection in $L^{2}\left(\mathbb{R}^{3}\right) \otimes \mathbb{C}^{2}$ onto the lowest band of $H(B)$. Its integral kernel is

$$
\Pi(B)\left(x, x^{\prime}\right)
$$

$$
=\frac{|B|}{2 \pi} \exp \left(i\left(x_{\perp} \wedge x_{\perp}^{\prime}\right) \frac{B}{2}-\left(x_{\perp}-x_{\perp}^{\prime}\right)^{2} \frac{|B|}{4}\right) \delta\left(x_{3}-x_{3}^{\prime}\right) \mathcal{P}^{\downarrow}
$$

in coordinates $x=\left(x_{\perp}, x_{3}\right)$ where $B=(0,|B|)$, and $\mathcal{P}^{\downarrow}=\left(1+\sigma_{3}\right) / 2$ is the projection in $\mathbb{C}^{2}$ onto the subspace where $B \cdot \sigma=|B|$. We remark that $\widehat{\Pi}$ commutes with $\widehat{H}^{0}$. The operator appearing on the right hand side of $(3.8)$ is then split as $\left(\widehat{H}^{0}+E\right)^{-1 / 2} J(V-E)_{+}^{1 / 2}=K_{0}(E)+K_{1}(E)$, with

$$
\begin{gathered}
K_{0}(E)=\left(\widehat{H}^{0}+E\right)^{-1 / 2} \widehat{\Pi} J(V-E)_{+}^{1 / 2}, \\
K_{1}(E)=\left(\widehat{H}^{0}+E\right)^{-1 / 2}(1-\widehat{\Pi}) J(V-E)_{+}^{1 / 2},
\end{gathered}
$$

so that by (3.2) it suffices to estimate $n\left(K_{i}(E)\right.$, const), $i=0,1$, separately. The first term is bounded by

$$
\begin{aligned}
& n\left(K_{0}(E), \text { const }\right) \lesssim \operatorname{tr} K_{0}(E)^{*} K_{0}(E) \\
&=\int d^{3} y \operatorname{tr}\left(j_{y}(V-E)_{+}^{1 / 2} \Pi\left(B_{y}\right)\left(H\left(B_{y}\right)+E\right)^{-1}\right. \\
&\left.\cdot \Pi\left(B_{y}\right)(V-E)_{+}^{1 / 2} j_{y}\right) \\
&=\left(4 \pi E^{1 / 2}\right)^{-1} \int d^{3} y d^{3} x(V(x)-E)_{+} j_{y}(x)^{2}\left|B_{y}\right|
\end{aligned}
$$

where the last estimate is $[10,(2.15)]$. Note that the gauge transformation $e^{i f_{y}}$ disappeared from the trace by cyclicity. For the second term we use the inequality before $[10,(2.18)]$, which states that 
$3 H\left(B_{y}\right) / 2 \geq D_{y}^{2} \equiv\left(p-(1 / 2) B_{y} \wedge x\right)^{2}$ on the orthogonal complement Ran $\left(1-\Pi\left(B_{y}\right)\right)$ of the lowest Landau band. We hence get

$$
\widehat{H}^{0} \geq \frac{2}{3} \int_{\mathbb{R}^{3}}^{\oplus} e^{i f_{y}} D_{y}^{2} e^{-i f_{y}} d^{3} y \equiv \widehat{H}_{S}
$$

on Ran $(1-\widehat{\Pi})$, as well as $(1-\widehat{\Pi})\left(\widehat{H}^{0}+E\right)^{-1}(1-\widehat{\Pi}) \leq\left(\widehat{H}_{S}+E\right)^{-1}$, because $\widehat{\Pi}$ and $\widehat{H}_{S}$ commute. Together with $n(X, 1) \leq \operatorname{tr}\left(\left(X^{*} X\right)^{2}\right)$ this yields

$$
\begin{aligned}
& n\left(K_{1}(E), \text { const }\right) \\
& \lesssim \operatorname{tr}\left((V-E)_{+}^{1 / 2} J^{*}\left(\widehat{H}_{S}+E\right)^{-1} J(V-E)_{+}\right. \\
& \left.\quad \cdot J^{*}\left(\widehat{H}_{S}+E\right)^{-1} J(V-E)_{+}^{1 / 2}\right) \\
& =\int \operatorname{tr}\left(j_{y} j_{y^{\prime}} e^{i\left(f_{y}-f_{y^{\prime}}\right)}(V-E)_{+}\left(\frac{2}{3} D_{y}^{2}+E\right)^{-1}\right. \\
& \left.\quad \cdot j_{y} j_{y^{\prime}} e^{-i\left(f_{y}-f_{y^{\prime}}\right)}(V-E)_{+}\left(\frac{2}{3} D_{y^{\prime}}^{2}+E\right)^{-1}\right) d^{3} y d^{3} y^{\prime} .
\end{aligned}
$$

Using the pointwise diamagnetic inequality [16] for the resolvent kernel

$$
\left|\left(\frac{2}{3} D_{y}^{2}+E\right)^{-1}\left(x, x^{\prime}\right)\right| \leq\left(\frac{2}{3} p^{2}+E\right)^{-1}\left(x-x^{\prime}\right),
$$

the trace under the integral is bounded as in (I.3.9) by

$$
\frac{3}{8 \pi}\left(\frac{3}{2 E}\right)^{1 / 2} \int(V(x)-E)_{+}^{2} j_{y}(x)^{2} j_{y^{\prime}}(x)^{2} d^{3} x
$$

This leads to $n\left(K_{1}(E)\right.$, const $) \lesssim E^{-1 / 2} \int(V(x)-E)_{+}^{2} d^{3} x$ by $(2.11)$ and, together with (3.10), to

$$
\begin{aligned}
\int_{0}^{\infty} n\left(K_{>}(E)\right. & \left., \frac{1}{2}\right) d E \\
& \lesssim \int d^{3} x V(x)^{3 / 2}\left(V(x)+\int d^{3} y\left|B_{y}\right| j_{y}(x)^{2}\right) .
\end{aligned}
$$

We now turn to $K_{<}$. The inequality

$$
\int_{0}^{\infty} n\left(K_{<}(E), \frac{1}{2}\right) d E \lesssim \varepsilon^{-6} \operatorname{tr}\left(V^{1 / 2} P J^{*} \widehat{H}^{-2} J P V^{1 / 2}\right)
$$


follows from (3.3), from $\int_{0}^{\infty} n\left(X, \mu^{1 / 2}\right) d \mu=\operatorname{tr} X^{*} X$, and from (3.4). We then split $\widehat{H}^{-2}=\widehat{\Pi} \widehat{H}^{-2} \widehat{\Pi}+(1-\widehat{\Pi}) \widehat{H}^{-2}(1-\widehat{\Pi})$. The contribution of the first term is

$$
\begin{array}{r}
\int d^{3} y \operatorname{tr}\left(j_{y} V^{1 / 2} P \Pi\left(B_{y}\right)\left(H\left(B_{y}\right)+\varepsilon^{-3} P(y)\right)^{-2} \Pi\left(B_{y}\right) P V^{1 / 2} j_{y}\right) \\
=\frac{1}{8 \pi} \int\left(\varepsilon^{-3} P(y)\right)^{-3 / 2}\left|B_{y}\right| P(x)^{2} V(x) j_{y}(x)^{2} d^{3} y d^{3} x
\end{array}
$$

because of $(3.9)$ and of $\Pi(B)(H(B)+E)^{-2}=\Pi(B)\left(p_{3}^{2}+E\right)^{-2}$ in the coordinates used there. For the second term we use (see (3.11)) $\widehat{H}^{2} \geq\left(\widehat{H}_{S}+\widehat{P}\right)^{2}$ on Ran $(1-\widehat{\Pi})$, since $\widehat{H}$ and $\widehat{H}_{S}+\widehat{P}$ commute, where $\widehat{P}=\varepsilon^{-3} \int_{\mathbb{R}^{3}}^{\oplus} P(y) d^{3} y$. This yields a contribution bounded by

$$
\begin{aligned}
\int \operatorname{tr}\left(j_{y} V^{1 / 2}\right. & \left.P\left(\frac{2}{3} D_{y}^{2}+\varepsilon^{-3} P(y)\right)^{-2} P V^{1 / 2} j_{y}\right) d^{3} y \\
& \leq \frac{3}{8 \pi} \int\left(\frac{3}{2 \varepsilon^{-3} P(y)}\right)^{1 / 2} P(x)^{2} V(x) j_{y}(x)^{2} d^{3} y d^{3} x
\end{aligned}
$$

where we used again (3.12). Taking into account (2.14) and (2.11) we thus obtain

$$
\begin{aligned}
& \int_{0}^{\infty} n\left(K_{<}(E), \frac{1}{2}\right) d E \\
& \quad \stackrel{(3.14)}{\quad \lesssim \int d^{3} x V(x)\left(\varepsilon^{-9 / 2} P(x)^{3 / 2}+\varepsilon^{-3 / 2} P(x)^{1 / 2} \int d^{3} y\left|B_{y}\right| j_{y}(x)^{2}\right) .}
\end{aligned}
$$

In order to put the result, i.e., the sum of (3.13) and (3.14), into the form given in Theorem 1 we estimate

$$
\left|B_{y}\right| \leq\left|K_{y}\right|^{-1} \int_{K_{y}}|B(z)| d^{3} z=\left|K_{y}\right|^{-1} \int|B(z)| \theta(|z-y|<2 \varepsilon l(y)) d^{3} z
$$

where $\theta(A)$ is the characteristic function of the set $A$, so that

$$
\int d^{3} y\left|B_{y}\right| j_{y}(x)^{2}
$$

$$
\leq \int d^{3} z|B(z)| \int d^{3} y\left|K_{y}\right|^{-1} \theta(|z-y|<2 \varepsilon l(y)) j_{y}(x)^{2} .
$$


We recall that $\operatorname{supp} j_{y} \subset\{x:|x-y| \leq \varepsilon l(x)\}$. Using again (2.10) and the triangle inequality $|x-z| \leq|x-y|+|z-y|$ we bound (3.15) by a constant times

$$
\begin{aligned}
\left|K_{x}\right|^{-1} \int d^{3} z|B(z)| \theta(|x-z|< & 5 \varepsilon l(x)) \int d^{3} y j_{y}(x)^{2} \\
& =\left|K_{x}\right|^{-1} \int_{|x-z|<5 \varepsilon l(x)} d^{3} z|B(z)|,
\end{aligned}
$$

i.e., by $\widehat{B}(x)$ after a redefinition of $\varepsilon$.

At this point Theorem 1 is proven, except for Lemma 6 and (3.3).

\section{Proofs.}

In this section we give all the proofs we omitted in the previous one in order to complete the derivation of (1.5).

Lemma 7. Let $U \in L^{3 / 2}\left(\mathbb{R}^{3}\right)$. Then

$$
U \leq \frac{1}{3}\left(\frac{\pi}{2}\right)^{-4 / 3}\|U\|_{3 / 2} D^{2}
$$

For a proof, see Lemma I.7 and subsequent remark.

\section{Lemma 8.}

$$
D l^{-2} D \lesssim \not D^{2} P+P \not D^{2}+\varepsilon^{-2} P^{2}
$$

Proof. The first step towards (4.2) consists in showing

$$
D l^{-2} D \lesssim \not D^{2} l^{-2}+l^{-2} \not D^{2}+\varepsilon^{-2} P^{2}
$$

This statement is closely related to Lemma I.8 and, similarly, its proof reduces to that of

$$
l^{-2}|B| \lesssim \varepsilon^{1 / 2}\left(D l^{-2} D+\varepsilon^{-2} P^{2}\right) .
$$

This is again proven as in I, except for the fact that we use here (and only here) a partition of unity based on the length scale $\varepsilon \lambda(x)$ as discussed at the end of Section 2, with $\lambda(x)^{-1}=r(x)^{-1}+l(x)^{-1}$. In 
particular, we now set $\widetilde{K}_{y}=\{x:|x-y|<\varepsilon \lambda(x)\}$ with characteristic function $\tilde{\chi}_{y}$. It then still holds that

$$
\begin{aligned}
\left\|l^{-2}|B| \tilde{\chi}_{y}\right\|_{3 / 2} & \leq\left\|l^{-2} \tilde{\chi}_{y}\right\|_{\infty}\left\|B \tilde{\chi}_{y}\right\|_{2}\left\|\tilde{\chi}_{y}\right\|_{6} \\
& \lesssim l(y)^{-2} r(y)^{-1 / 2}(\varepsilon r(y))^{1 / 2} \\
& =\varepsilon^{1 / 2} l(y)^{-2}
\end{aligned}
$$

where: we used $\lambda(x) \leq l(x)$ in estimating the first factor; $\lambda(x) \leq r(x)$ and (2.1) in the second; and again $\lambda(x) \leq r(x)$ in the last one. We hence obtain, just as in I,

$$
l^{-2}|B| \lesssim \varepsilon^{1 / 2}\left(D l^{-2} D+l^{-2} \int\left(\nabla j_{y}\right)^{2} d^{3} y\right)
$$

with the integral bounded by $(\varepsilon \lambda(x))^{-2}$ due to (2.12). The proof of (4.4), and hence of (4.3), is completed by noticing that $l^{-2} \lambda^{-2}=P^{2}$. We now come back to (4.2). We have

$$
\pm\left(\not D^{2} f+f \not D^{2}-2 \not D f \not D\right) \lesssim \varepsilon \not D P \not D+\varepsilon^{-1} P^{2},
$$

for $f=l^{-2}$ or $f=P$. Indeed, the left hand side is

$$
\pm[\not D,[\not D, f]]=\mp i[\not D, \nabla f \cdot \sigma]=-X^{*} X+\varepsilon \not D P \not D+\varepsilon^{-1} P^{-1}(\nabla f)^{2}
$$

with $X=(\varepsilon P)^{1 / 2} \not D \pm i(\varepsilon P)^{-1 / 2} \nabla f \cdot \sigma$ and $(\nabla f)^{2} \lesssim P^{3}$ due to $(2.7)$ respectively (2.13). Taking $f=l^{-2}$ we first obtain from (4.3)

$$
D l^{-2} D \lesssim \not D l^{-2} \not D+\varepsilon \not D P \not D+\varepsilon^{-1} P^{2}+\varepsilon^{-2} P^{2} \leq 2\left(\not D P \not D+\varepsilon^{-2} P^{2}\right),
$$

and then, with $f=P$, we obtain (4.2).

Proof OF (3.5). The localization argument begins as that given for (I.3.2), with $b$ replaced by $P$, i.e., we have

$$
\not D^{4}=\int\left(j_{y} \not D^{4} j_{y}+\frac{1}{2}\left(\left[j_{y},\left[j_{y}, \not D^{2}\right]\right], \not D^{2}\right)+\left[j_{y}, \not D^{2}\right]^{2}\right) d^{3} y,
$$

with the estimate

$$
-\int \frac{1}{2}\left(\left[j_{y},\left[j_{y}, \not D^{2}\right]\right], \not D^{2}\right) d^{3} y \leq \frac{1}{2} \varepsilon^{-3}\left(\not D^{2} P+P \not D^{2}\right)+\varepsilon^{-5} P^{2}
$$


for the first localization error. The other one is estimated similarly

$$
\begin{aligned}
-\int\left[j_{y}, \not D^{2}\right]^{2} d^{3} y & \leq \operatorname{const}\left(\varepsilon^{-2} D l^{-2} D+\varepsilon^{-4} l^{-4}\right) \\
& \leq \frac{1}{2} \varepsilon^{-3}\left(\not D^{2} P+P \not D^{2}\right)+\varepsilon^{-5} P^{2},
\end{aligned}
$$

by using (4.2). The conclusion then is as in I.

Lemma $9([7])$. Let $K=\{x:|x|<1\}$ be the unit ball, and $K^{*}=2 K$. Let $B \in L^{2}\left(K^{*}, \mathbb{R}^{3}\right)$ be a vector field with $\nabla \cdot B=0$ (as a distribution) and

$$
\int_{K} B(x) d^{3} x=0
$$

Then there is a vector field $A$ such that

$$
\nabla \wedge A=B, \quad \nabla \cdot A=0
$$

and

$$
\|A\|_{\infty, K} \lesssim\|\nabla \otimes B\|_{2, K^{*}}
$$

Proof. A solution $A$ to (4.6) is constructed as in I, i.e., as $A=\nabla \wedge F$, where $F$ is the solution of $-\Delta F=B$ with boundary conditions (I.4.11). By $\|F\|_{2, K^{*}} \lesssim\|B\|_{2, K^{*}}$ and the elliptic estimate

$$
\left\|\nabla^{\otimes 3} F\right\|_{2, K} \lesssim\|F\|_{2, K^{*}}+\|\Delta F\|_{2, K^{*}}+\|\nabla \otimes \Delta F\|_{2, K^{*}}
$$

we have

$$
\left\|\nabla^{\otimes 2} A\right\|_{2, K} \lesssim\|B\|_{2, K^{*}}+\|\nabla \otimes B\|_{2, K^{*}} \lesssim\|\nabla \otimes B\|_{2, K^{*}} .
$$

In establishing the last inequality we used that a Poincaré inequality (see e.g. [20, Theorem 4.4.2]) applies to $\|B\|_{2, K^{*}}$, due to (4.5). Another Poincaré type inequality ([20, Corollary 4.2.3]) yields

$$
\|A-\alpha-\beta x\|_{\infty, K} \lesssim\left\|\nabla^{\otimes 2} A\right\|_{2, K},
$$

for $\alpha_{i}=|K|^{-1} \int_{K} A_{i}(x) d^{3} x$ and $\beta_{i j}=|K|^{-1} \int_{K} \partial_{j} A_{i}(x) d^{3} x$. This proves (4.7) for $A-\alpha-\beta x$ instead of $A$. Equation (4.6) is preserved under this replacement, since it implies $\beta_{i j}-\beta_{j i}=0$ and $\operatorname{tr} \beta=0$. 
Proof of (3.6). Let $B_{y}=\left|K_{y}\right|^{-1} \int_{K_{y}} B(x) d^{3} x$ be the average magnetic field over $K_{y}=\{x:|x-y|<2 \varepsilon l(y)\}$. It is generated by the vector potential $A_{y}(x)=(1 / 2) B_{y} \wedge(x-y)$. On the other hand, let $\widetilde{A}_{y}(x)$ be the vector potential of $\widetilde{B}_{y}(x)=B(x)-B_{y}$, which by scaling corresponds to the one constructed in the previous lemma. It satisfies

$$
\left|\widetilde{A}_{y}(x)\right| \lesssim \varepsilon^{1 / 2} l(y)^{-1},
$$

for $x \in K_{y}$ because of (2.2), (4.7). Since $B=\nabla \wedge\left(A_{y}+\widetilde{A}_{y}\right)$, we may assume, upon making a gauge transformation, $A=A_{y}+\widetilde{A}_{y}$. The Pauli operators corresponding to $\not D_{y}=\left(p-A_{y}\right) \cdot \sigma$ and $\not D$ are related as

$$
\begin{aligned}
\not D_{y}^{2}=\left(\not D+\widetilde{A}_{y} \cdot \sigma\right)^{2} & =\not D^{2}+\left(\widetilde{A}_{y}\right)^{2}+\left\{\widetilde{A}_{y} \cdot \sigma, \not D\right\} \\
& =\not D^{2}+\left(\widetilde{A}_{y}\right)^{2}+\left\{\widetilde{A}_{y}, D\right\}+\widetilde{B}_{y} \cdot \sigma .
\end{aligned}
$$

This and $\nabla \cdot \widetilde{A}_{y}=0$ yield

$$
\not D_{y}^{4} \leq 4\left(\not D^{4}+\left(\widetilde{A}_{y}\right)^{4}+4 D\left(\widetilde{A}_{y}\right)^{2} D+\left(\widetilde{B}_{y}\right)^{2}\right) .
$$

After multiplying from both sides with $j_{y}$ we may replace $\widetilde{A}_{y}$ by $\chi_{y} \widetilde{A}_{y}$ and similarly for $\widetilde{B}_{y}$, where $\chi_{y}(x)$ is the characteristic function of $K_{y}$. Note that, besides of (4.8), we have by (2.2) and $\left\|\chi_{y}\right\|_{3} \lesssim \varepsilon l(y)$

$$
\left\|\widetilde{B}_{y}^{2} \chi_{y}\right\|_{3 / 2} \leq\left\|\widetilde{B}_{y}^{2} \chi_{y}\right\|_{3}\left\|\chi_{y}\right\|_{3} \lesssim\left\|(\nabla \otimes B)^{2} \chi_{y}\right\|_{1}\left\|\chi_{y}\right\|_{3} \lesssim \varepsilon l(y)^{-2} .
$$

We can thus estimate, using (4.1),

$$
j_{y} \not D_{y}^{4} j_{y} \lesssim j_{y}\left(\not D^{4}+\varepsilon^{2} l(y)^{-4}+\varepsilon D l(y)^{-2} D\right) j_{y}
$$

and finally, using (2.10), (2.14), (4.2),

$$
\begin{aligned}
j_{y}\left(\not D_{y}^{2}+\varepsilon^{-3} P(y)\right)^{2} j_{y} & \leq 2 j_{y}\left(\not D_{y}^{4}+\varepsilon^{-6} P(y)^{2}\right) j_{y} \\
& \lesssim j_{y}\left(\not D^{4}+\frac{1}{2} \varepsilon^{-6} P(x)^{2}+\varepsilon D l(x)^{-2} D\right) j_{y} \\
& \leq j_{y}\left(\not D^{2}+\varepsilon^{-3} P\right)^{2} j_{y} .
\end{aligned}
$$


Proof OF (3.3). The proof can be taken over literally from that of (I.3.6), after replacing $b$ by $P$. To be checked however is that $f=\log P$ satisfies $(\nabla f)^{2} \lesssim l^{-2} \leq P$ and $|\Delta f| \lesssim P$, as well as $D(\nabla f)^{2} D \lesssim$ $\not D^{2} P+P \not D^{2}+\varepsilon^{-2} P^{2}$. This follows from (2.13), (4.2).

\section{Stability of matter.}

As an application of (1.5), we state and prove a stability estimate for matter coupled to a classical magnetic field. It is essentially identical to a result of [7], except for exhibiting a somewhat more explicit dependence of the stability bound on the parameters involved. The system we consider consists of $N$ spin $1 / 2$ electrons (with Hilbert space $\left.\wedge^{N} \mathcal{H}, \mathcal{H}=L^{2}\left(\mathbb{R}^{3}\right) \otimes \mathbb{C}^{2}\right)$ interacting with $K$ static nuclei, having positions $R_{k}$ and charges $Z>0$, and with a classical magnetic field $B$. The theorem then reads:

Theorem 10. Let $\mathcal{R}=\left\{R_{k}\right\}_{k=1}^{K}$ and $R, Z, \Gamma, \gamma>0$. There is $C(Z, \Gamma, \gamma)$ and a function $\Phi_{\mathcal{R}}(x) \geq 0$ with

$$
\left\|\Phi_{\mathcal{R}}\right\|_{\infty} \lesssim 1, \quad\left\|\Phi_{\mathcal{R}}\right\|_{1} \lesssim R^{3} K
$$

uniformly in $\mathcal{R}, Z$, such that the $N$-body Hamiltonian

$$
\begin{aligned}
H_{N}= & \sum_{i=1}^{N} \not D_{i}^{2}+V_{C} \\
& +\Gamma \int \Phi_{\mathcal{R}}(x)\left(B(x)^{2}+\gamma R^{2}(\nabla \otimes B)(x)^{2}\right) d^{3} x \\
V_{C}= & \sum_{\substack{i, j=1 \\
i<j}}^{N} \frac{1}{\left|x_{i}-x_{j}\right|}-\sum_{i, k=1}^{N, K} \frac{Z}{\left|x_{i}-R_{k}\right|}+\sum_{\substack{k, l=1 \\
k<l}}^{K} \frac{Z^{2}}{\left|R_{k}-R_{l}\right|},
\end{aligned}
$$

acting on $\wedge^{N} \mathcal{H}$, satisfies

$$
H_{N} \geq-C(Z, \Gamma, \gamma)(Z+1) R^{-1}(N+K)
$$

for arbitrary $R \leq(Z+1)^{-1}$. For $\Gamma \leq Z+1$ and $1 \leq \gamma \leq z^{4}$ one can take

$$
C(Z, \Gamma, \gamma)=\operatorname{const}\left(z^{3}+z^{5} \gamma^{-1 / 2} \log \left(z^{5} \gamma^{-1 / 2}\right)\right)
$$


with $z=1+(Z+1) \Gamma^{-1}$.

REMARK. One may modify the definition (2.2) of $l(x)$ by replacing $(\nabla \otimes B)^{2}$ by $(\nabla \otimes B)^{2}+R^{-6}$ for some $R>0$. Theorem 1 continues to hold. On the right hand side of (2.6) a term $R^{-6}$ should also be added to $(\nabla \otimes B)^{2}$, but it can be absorbed into the last term. The purpose of this variant is to ensure

$$
l(x) \lesssim R
$$

Proof. By monotonicity, it will be enough to prove the theorem for $Z \geq 1, \Gamma \leq Q$ and $\gamma \leq z^{4}$. We partition [9] $\mathbb{R}^{3}$ into Voronoi cells $\Gamma_{j}=\left\{x:\left|x-R_{j}\right| \leq\left|x-R_{k}\right|\right.$ for $\left.k=1, \ldots, K\right\}, j=1, \ldots, K$. Let $D_{j}=\min \left\{\left|R_{j}-R_{k}\right|: j \neq k\right\} / 2$. For any $\nu>0$ the reduction to a one-body problem reads [9], [12]

$$
\begin{aligned}
H_{N} \geq & \sum_{i=1}^{N} h_{i}-\nu N+\frac{Z^{2}}{8} \sum_{j=1}^{K} D_{j}^{-1} \\
& +\Gamma \int \Phi_{\mathcal{R}}(x)\left(B(x)^{2}+\gamma R^{2}(\nabla \otimes B)(x)^{2}\right) d^{3} x,
\end{aligned}
$$

where $h=\not D^{2}-(W-\nu)_{+}$and $W$ is a potential satisfying $W(x) \leq$ $Q\left|x-R_{j}\right|^{-1}$ for $x \in \Gamma_{j}$, with $Q=Z+\sqrt{2 Z}+2.2$.

We choose $\nu=Q R^{-1}$ and apply Theorem 1 (in the variant discussed above) to obtain

$$
\begin{aligned}
\sum_{i=1}^{N} h_{i} \gtrsim & -\int V^{5 / 2} d^{3} x-\int P^{3 / 2} V d^{3} x \\
& -\int \widehat{B} V^{3 / 2} d^{3} x-\int \widehat{B} P^{1 / 2} V d^{3} x,
\end{aligned}
$$

where $V=\left(W-Q R^{-1}\right)_{+}$. Comparing with (5.6) it appears to be enough to show that each of the integrals (5.7), which we shall denote by i)-iv) below, is bounded by the bound (5.3) or by a small (universal) constant times

$$
\frac{Z^{2}}{8} \sum_{j=1}^{K} D_{j}^{-1}+\Gamma \int \Phi_{\mathcal{R}}(x)\left(B(x)^{2}+\gamma R^{2}(\nabla \otimes B)(x)^{2}\right) d^{3} x
$$


i) Note that $\operatorname{supp} V \subset \Omega_{R}$ for $\Omega=\left\{R_{j}: j=1, \ldots, K\right\}$. This integral is thus bounded by const $Q^{5 / 2} R^{1 / 2} K \lesssim Q R^{-1} K$.

ii) We note that for any $\beta_{1}>0$

$$
P^{3 / 2} \leq \sqrt{2} l^{-3 / 2}\left(r^{-3 / 2}+l^{-3 / 2}\right) \leq \sqrt{2} \frac{\beta_{1}}{2} r^{-3}+\sqrt{2}\left(1+\frac{\beta_{1}^{-1}}{2}\right) l^{-3}
$$

and we estimate the contributions to ii) of the two terms separately. For the first one we use that

$$
\int_{\Omega_{R}} r(x)^{-3} V(x) d^{3} x \lesssim Q \int \Phi_{\mathcal{R}}(x) B(x)^{2} d^{3} x+Q \sum_{j=1}^{K} D_{j}^{-1}+Q R^{-1} K
$$

as was shown in Section I.5. This is consistent with the bound (5.3) if $\beta_{1} \ll \min \left\{Q^{-1} \Gamma, 1\right\}$. (By $a \ll b$ we mean $a=$ const $b$ for some sufficiently small universal constant). For the last term in (5.9) we use instead

$$
\begin{aligned}
\int_{\Omega_{R}} l(x)^{-3} V(x) d^{3} x & \\
& \leq \frac{\beta_{2}}{2} \int_{\Omega_{R}} l(x)^{-6} d^{3} x+\frac{\beta_{2}^{-1}}{2} \int_{\Omega_{R}} V(x)^{2} d^{3} x \\
& \lesssim \beta_{2} \int \Phi_{\mathcal{R}}(x)(\nabla \otimes B)(x)^{2} d^{3} x+\left(\beta_{2} R^{-3}+\beta_{2}^{-1} Q^{2} R\right) K
\end{aligned}
$$

due to (2.6). The desired bound holds provided we pick $z \cdot \beta_{2} \ll \Gamma \gamma R^{2}$.

iii) We split the integral into $K$ inner integrals over $U_{j}=\{x$ : $\left.\left|x-R_{j}\right| \leq \widehat{D}_{j}\right\}, \widehat{D}_{j}=\min \left\{D_{j}, \varepsilon l\left(R_{j}\right), R\right\}$ for some small $\varepsilon>0$; and one outer integral over $\mathbb{R}^{3} \backslash \bigcup_{j=1}^{K} U_{j}$. The inner integrals can be estimated as

$$
\begin{aligned}
\int_{U_{j}} \widehat{B}(x) V(x)^{3 / 2} d^{3} x & \lesssim\left(\sup _{x \in U_{j}} \widehat{B}(x)\right) \widehat{D}_{j}^{3 / 2} Q^{3 / 2} \\
& \leq \frac{\beta}{2} \widehat{D}_{j}^{3}\left(\sup _{x \in U_{j}} \widehat{B}(x)^{2}\right)+\frac{\beta^{-1}}{2} Q^{3} .
\end{aligned}
$$


Because of (2.10) we have $l\left(R_{j}\right) / 2 \leq l(x) \leq 2 l\left(R_{j}\right)$ for $x \in U_{j}$ and thus

$$
\begin{aligned}
\widehat{B}(x)^{2} & =\left|K_{x}\right|^{-2}\left(\int_{K_{x}}|B(y)| d^{3} y\right)^{2} \\
& \leq\left|K_{x}\right|^{-1} \int_{K_{x}} B(y)^{2} d^{3} y \\
& \lesssim\left(\varepsilon l\left(R_{j}\right)\right)^{-3} \int \theta\left(\left|y-R_{j}\right| \leq 3 \varepsilon l\left(R_{j}\right)\right) B(y)^{2} d^{3} y .
\end{aligned}
$$

Altogether we find for any $\beta>0$

$$
\begin{gathered}
\int_{\cup_{j=1}^{K} U_{j}} \widehat{B}(x) V(x)^{3 / 2} d^{3} x \lesssim \beta \int \Phi(y) B(y)^{2} d^{3} y+\beta^{-1} Q^{3} K, \\
\Phi(y)=\sum_{j=1}^{K} \widehat{D}_{j}^{3}\left(\varepsilon l\left(R_{j}\right)\right)^{-3} \theta\left(\left|y-R_{j}\right| \leq 3 \varepsilon l\left(R_{j}\right)\right) .
\end{gathered}
$$

For $\beta \ll \Gamma$ this will be bounded as claimed once we show that

$$
\Phi \lesssim \theta_{\Omega_{R}}
$$

First, $\operatorname{supp} \Phi \subset \Omega_{R}$ for small $\varepsilon>0$ because of (5.5). It thus suffices to show $\|\Phi\|_{\infty} \lesssim 1$ : from $\widehat{D}_{j} \leq \varepsilon l\left(R_{j}\right)$, the triangle inequality and (2.10) we find

$$
\begin{gathered}
\|\Phi\|_{\infty} \leq \sup _{y} \sum_{j=1}^{K}\left(\varepsilon l\left(R_{j}\right)\right)^{-3} \theta\left(\left|y-R_{j}\right| \leq 3 \varepsilon l\left(R_{j}\right)\right) \\
\cdot \int_{U_{j}} \theta\left(\left|x-R_{j}\right| \leq \varepsilon l\left(R_{j}\right)\right) d^{3} x \\
\lesssim \sup _{y} \sum_{j=1}^{K}(\varepsilon l(y))^{-3} \int_{U_{j}} \theta(|x-y| \leq 8 \varepsilon l(y)) d^{3} x \\
\lesssim 1,
\end{gathered}
$$

since the $U_{j}$ are disjoint.

The outer integral can be written and estimated as

$$
\int_{\Omega_{R} \backslash\left(\cup_{j=1}^{K} U_{j}\right)} d^{3} x V(x)^{3 / 2}\left|K_{x}\right|^{-1} \int d^{3} y|B(y)| \theta(|x-y|<\varepsilon l(x))
$$




$$
\leq \frac{\beta_{1}}{2} \int_{\Omega_{R} \times \mathbb{R}^{3}} d^{3} x d^{3} y|B(y)|^{2}\left|K_{x}\right|^{-1} \theta(|x-y|<\varepsilon l(x))
$$

$$
+\frac{\beta_{1}^{-1}}{2} \int_{\Omega_{R} \backslash\left(\cup_{j=1}^{K} U_{j}\right) \times \mathbb{R}^{3}} d^{3} x d^{3} y V(x)^{3}\left|K_{x}\right|^{-1} \theta(|x-y|<\varepsilon l(x)) .
$$

By the usual argument (2.10), the first integral is bounded by a constant times $\int \Phi(y)|B(y)|^{2} d^{3} y$ for

$$
\Phi(y)=\left|K_{y}\right|^{-1} \int_{\Omega_{R}} \theta(|x-y|<2 \varepsilon l(y)) d^{3} x \lesssim 1 .
$$

Moreover, supp $\Phi \subset \Omega_{2 R}$ as before. It thus suffices to take $\beta_{1} \ll \Gamma$. In the second term on the right hand side of (5.11) the integration over $y$ is explicit, and the integral is

$$
\begin{aligned}
\int_{\Omega_{R} \backslash\left(\cup_{j=1}^{K} U_{j}\right)} V(x)^{3} d^{3} x & \lesssim \sum_{j=1}^{K} Q^{3} \log R \widehat{D}_{j}^{-1} \\
& \leq \beta_{2} Q^{3} \sum_{j=1}^{K} R \widehat{D}_{j}^{-1}+\left(\log \beta_{2}^{-1}\right) Q^{3} K
\end{aligned}
$$

where we used that $\log t \leq \beta_{2} t+\log \beta_{2}^{-1}$ for $t, \beta_{2}>0$. We shall take $\Gamma^{-1} \cdot \beta_{2} Q^{2} R \ll 1$, so that the last term is of the desired form. The first one reduces to an arbitrarily small constant times $Q \sum_{j=1}^{K} \widehat{D}_{j}^{-1}$. Note that

$$
\widehat{D}_{j}^{-1} \lesssim \varepsilon^{-2}\left(\int_{U_{j}} l(x)^{-6} d^{3} x\right)^{1 / 3}+D_{j}^{-1}+R^{-1}
$$

In fact, by (2.10), the integral is bounded below by a constant times $\left(\varepsilon l\left(R_{j}\right)\right)^{-2} \widehat{D}_{j}$, and thus the whole right hand side by

$$
\widehat{D}_{j}^{-1}\left(\left(\frac{\widehat{D}_{j}}{\varepsilon l\left(R_{j}\right)}\right)^{2}+\frac{\widehat{D}_{j}}{D_{j}}+\frac{\widehat{D}_{j}}{R}\right) \geq \widehat{D}_{j}^{-1},
$$

by definition of $\widehat{D}_{j}$. The contribution of the last two terms of (5.13) are then controlled by the first term (5.8), respectively by (5.3). For the integral, $I$, we use $I^{1 / 3} \leq 2 \beta_{3}^{-1 / 2} / 3+\beta_{3} I / 3$ and choose $Q \cdot \beta_{3} \varepsilon^{-2} \ll$ 
$\Gamma z^{-4} R^{2}$. Note that the $U_{j}$ are disjoint, allowing for the application of (2.6).

iv) Using

$$
P^{1 / 2} \leq l^{-1 / 2}\left(r^{-1 / 2}+l^{-1 / 2}\right) \leq \frac{\beta_{1}}{2} r^{-1}+\left(1+\frac{\beta_{1}^{-1}}{2}\right) l^{-1},
$$

we estimate the contributions to iv) of the two terms separately. The first integral is

$$
\begin{aligned}
& \int_{\Omega_{R}} d^{3} x r(x)^{-1} V(x)\left|K_{x}\right|^{-1} \int d^{3} y|B(y)| \theta(|x-y|<\varepsilon l(x)) \\
& \leq \frac{Q}{2} \int_{\Omega_{R} \times \mathbb{R}^{3}} d^{3} x d^{3} y|B(y)|^{2}\left|K_{x}\right|^{-1} \theta(|x-y|<\varepsilon l(x))
\end{aligned}
$$

$$
+\frac{Q^{-1}}{2} \int d^{3} x d^{3} y r(x)^{-2} V(x)^{2}\left|K_{x}\right|^{-1} \theta(|x-y|<\varepsilon l(x)) .
$$

The first term on the right hand side is like the corresponding one in (5.11) and hence acceptable provided $\beta_{1} \cdot Q \ll \Gamma$. The second integral, $Q^{-1} \int r(x)^{-2} V(x)^{2} d^{3} x$, is dealt with by splitting it with respect to $\widetilde{U}_{j}=$ $\left\{x:\left|x-R_{j}\right|<\widetilde{D}_{j}\right\}, \widetilde{D}_{j}=\min \left\{D_{j}, \varepsilon r\left(R_{j}\right), R\right\}$ (see Section I.5). Then

$$
\int_{\widetilde{U}_{j}} r(x)^{-2} V(x)^{2} d^{3} x \lesssim r\left(R_{j}\right)^{-2} \int_{\widetilde{U}_{j}} V(x)^{2} d^{3} x \lesssim \varepsilon^{2} Q^{2} \widetilde{D}_{j}^{-1},
$$

and

$$
\begin{aligned}
\int_{\mathbb{R}^{3} \backslash\left(\cup_{j=1}^{K} \widetilde{U}_{j}\right)} r(x)^{-2} V(x)^{2} d^{3} x & \\
& \leq \frac{\varepsilon^{2} Q^{-2}}{2} \int_{\mathbb{R}^{3} \backslash\left(\cup_{j=1}^{K} \widetilde{U}_{j}\right)} V(x)^{4} d^{3} x+\frac{\varepsilon^{-2} Q^{2}}{2} \int_{\Omega_{R}} r(x)^{-4} d^{3} x .
\end{aligned}
$$

Since the first integral is bounded above by const $Q^{4} \sum_{j=1}^{K} \widetilde{D}_{j}^{-1}$ we have that

$$
\begin{aligned}
Q^{-1} \int r(x)^{-2} V(x)^{2} d^{3} x & \\
& \lesssim Q \sum_{j=1}^{K} \widetilde{D}_{j}^{-1}+Q \int_{\Omega_{R}} r(x)^{-4} d^{3} x \\
& \lesssim Q \sum_{j=1}^{K} D_{j}^{-1}+Q \int \Phi_{\mathcal{R}}(x) B(x)^{2} d^{3} x+Q R^{-1} K
\end{aligned}
$$


due (I.5.4) (augmented by $R^{-1}$ ) and (2.5). These terms fit (5.3) for our choice of $\beta_{1}$.

The integral corresponding to the last term in (5.14) is estimated similarly to iii) and is split accordingly. The inner integrals can be estimated as

$$
\begin{aligned}
\int_{U_{j}} \widehat{B}(x) l(x)^{-1} V(x) d^{3} x \\
\quad \lesssim\left(\sup _{x \in U_{j}} \widehat{B}(x) l(x)^{-1}\right) \widehat{D}_{j}^{2} Q \\
\leq \frac{2 \beta_{2}^{1 / 2}}{3} \widehat{D}_{j}^{3}\left(\sup _{x \in U_{j}} \widehat{B}(x) l(x)^{-1}\right)^{3 / 2}+\frac{\beta_{2}^{-1}}{3} Q^{3}
\end{aligned}
$$

where

$$
\left(\widehat{B} l^{-1}\right)^{3 / 2} \leq \frac{1}{4} \gamma^{-1 / 4} R^{-1 / 2}\left(3 \widehat{B}^{2}+\gamma R^{2} l^{-6}\right) .
$$

The term coming from $\widehat{B}^{2}$ will be dealt with by (5.10), the other one by using

$$
\widehat{D}_{j}^{3} \sup _{x \in U_{j}} l(x)^{-6} \lesssim \int_{U_{j}} l(x)^{-6} d^{3} x .
$$

Choosing $z \cdot \beta_{2}^{1 / 2} \gamma^{-1 / 4} R^{-1 / 2} \ll \Gamma$ ensures that both terms (5.17) are controlled by (5.8) and (5.3). The contribution of the last term (5.16) is then of order $z \cdot \beta_{2}^{-1} Q^{3} K \lesssim z^{5} \gamma^{-1 / 2} Q R^{-1} K$. The estimate of the outer integral follows the line of (5.15)

$$
\begin{gathered}
\int_{\Omega_{R} \backslash\left(\cup_{j=1}^{K} U_{j}\right)} d^{3} x l(x)^{-1} V(x)\left|K_{x}\right|^{-1} \int d^{3} y|B(y)| \theta(|x-y|<\varepsilon l(x)) \\
\leq \frac{\beta_{3}}{2} \int_{\Omega_{R} \times \mathbb{R}^{3}} d^{3} x d^{3} y|B(y)|^{2}\left|K_{x}\right|^{-1} \theta(|x-y|<\varepsilon l(x)) \\
+\frac{\beta_{3}^{-1}}{2} \int_{\Omega_{R} \backslash\left(\cup_{j=1}^{K} U_{j}\right) \times \mathbb{R}^{3}} d^{3} x d^{3} y l(x)^{-2} V(x)^{2}\left|K_{x}\right|^{-1} \\
\cdot \theta(|x-y|<\varepsilon l(x)) .
\end{gathered}
$$

The first term just requires $z \beta_{3} \ll \Gamma$. The second one is

$$
\begin{aligned}
\int_{\mathbb{R}^{3} \backslash\left(\cup_{j=1}^{K} U_{j}\right)} l(x)^{-2} V(x)^{2} d^{3} x \\
\leq \frac{2}{3} \beta_{4}^{-1 / 2} \int_{\mathbb{R}^{3} \backslash\left(\cup_{j=1}^{K} U_{j}\right)} V(x)^{3} d^{3} x+\frac{1}{3} \beta_{4} \int_{\Omega_{R}} l(x)^{-6} d^{3} x .
\end{aligned}
$$


To accomodate the last term, after application of (2.6), we require $z^{2} \Gamma^{-1} \cdot \beta_{4} \ll \Gamma z^{-4} R^{2}$. The first term is dealt as in (5.12), with $\beta_{2} \ll z^{-7}$ there.

\section{Proof of Theorem 2.}

We split the total Hamiltonian into two parts [8], [2]

$$
H=H_{\mathrm{I}}+H_{\mathrm{II}}
$$

with

$$
\begin{gathered}
H_{\mathrm{I}}=\sum_{i=1}^{N} \not D_{i}^{2}+V_{C}+\Gamma \int \Phi_{\mathcal{R}}(x)\left(B(x)^{2}+\gamma R^{2}(\nabla \otimes B)(x)^{2}\right) d^{3} x, \\
H_{\mathrm{II}}=H_{f}-\Gamma \int \Phi_{\mathcal{R}}(x)\left(B(x)^{2}+\gamma R^{2}(\nabla \otimes B)(x)^{2}\right) d^{3} x,
\end{gathered}
$$

where $B=\nabla \wedge A$, and $\Phi_{\mathcal{R}}$ is the positive function appearing in Theorem 10. $\Gamma$ and $\gamma$ will be chosen later.

All the fields appearing in $H_{\mathrm{I}}$ are multiplication operators in the same Schrödinger representation of $\mathcal{F}$ [8]. Thus Theorem 10 applies and yields

$$
H_{\mathrm{I}} \geq-C(Z, \Gamma, \gamma)(Z+1) R^{-1}(N+K)
$$

We now turn to $H_{\mathrm{II}}$. Let $F(x)$ be either $B(x)$ or $\nabla \otimes B(x)$. As in (1.6), we may write $F(x)=F_{-}(x)+F_{+}(x)$ and obtain

$$
\begin{aligned}
F(x)^{2} & \leq F(x)^{2}+\left(F_{-}(x)-F_{+}(x)\right)^{*}\left(F_{-}(x)-F_{+}(x)\right) \\
& \leq 2\left(2 F_{+}(x) F_{-}(x)+\left[F_{-}(x), F_{+}(x)\right]\right)
\end{aligned}
$$

where the commutator is a multiple of the identity, independent of $x$. We then integrate against $f(x) d^{3} x$ with $f \geq 0$ and bound the first term using $f(x) \leq\|f\|_{\infty}$ and Parseval's identity. This yields

$$
\begin{aligned}
& \int f(x) B(x)^{2} d^{3} x \\
& \quad \leq 8 \pi \alpha\|f\|_{\infty} \int d^{3} k|k||\kappa(k)|^{2} \sum_{\lambda= \pm} a_{\lambda}(k)^{*} a_{\lambda}(k)+\frac{\alpha \Lambda^{4}}{\pi}\|f\|_{1},
\end{aligned}
$$


respectively

$$
\begin{aligned}
& \int f(x)(\nabla \otimes B)(x)^{2} d^{3} x \\
& \quad \leq 8 \pi \alpha\|f\|_{\infty} \int d^{3} k|k|^{3}|\kappa(k)|^{2} \sum_{\lambda= \pm} a_{\lambda}(k)^{*} a_{\lambda}(k)+\frac{2 \alpha \Lambda^{6}}{3 \pi}\|f\|_{1} .
\end{aligned}
$$

Note that the integrals on the right hand side are bounded by $\alpha H_{f}$ and $\alpha \Lambda^{2} H_{f}$, respectively. In particular, for $f=\Phi_{\mathcal{R}}$ we find

$$
\begin{aligned}
\Gamma \int \Phi_{\mathcal{R}}(x)\left(B(x)^{2}\right. & \left.+\gamma R^{2}(\nabla \otimes B)(x)^{2}\right) d^{3} x \\
\leq & \operatorname{const} \Gamma \alpha^{2}\left(1+\gamma(\Lambda R)^{2}\right)\left(H_{f}+\alpha^{-1} \Lambda^{4} R^{3} K\right) .
\end{aligned}
$$

We may now optimize over $\Gamma, \gamma, R$, within the ranges allowed by Theorem 10, in such a way that the factor in front of $H_{f}$ is less than 1. The resulting choice is as follows: We pick $\Gamma \ll Z^{*}\left(1+Z^{*} \alpha^{2}\right)^{-1}$ and $R=\gamma^{-1 / 2}\left(\Lambda+Z^{*}\left(Z^{*} \alpha^{2}\right)^{-2}\right)^{-1}$. As a result, the factor in front of $H_{\mathrm{f}}$ is indeed less than 1 and

$$
H_{\mathrm{II}} \gtrsim-Z^{*} \alpha \gamma^{-3 / 2} \Lambda K
$$

We finally choose $\gamma=z^{4}$ with $z$ as in Theorem 10 . Since $z \approx 1+Z^{*} \alpha^{2}$ we have $R \leq Z^{*-1}$, so that (6.1) applies

$$
\begin{aligned}
H_{\mathrm{I}} & \gtrsim-z^{3}(1+\log z) Z^{*} R^{-1}(N+K) \\
& \gtrsim-z^{5}(1+\log z) Z^{*}\left(\Lambda+Z^{*}\left(Z^{*} \alpha^{2}\right)^{-2}\right)(N+K) .
\end{aligned}
$$

This is also a lower bound to (6.2), because of $\alpha \leq 1+Z^{*} \alpha^{2}$.

Acknowledgements. We thank J. Fröhlich for very useful discussions. This work would not have been possible without his collaboration at an earlier stage.

\section{References.}

[1] Bugliaro, L., Fefferman, C., Fröhlich, J., Graf, G. M., Stubbe, J., A Lieb-Thirring Bound for a Magnetic Pauli Hamiltonian. Comm. Math. Phys. 187 (1997), 567-582. 
[2] Bugliaro, L., Fröhlich, J., Graf, G. M., Stability of quantum electrodynamics with non-relativistic matter. Phys. Rev. Lett. 77 (1996), 3494-3497.

[3] Dunford, N., Schwartz, J. T., Linear Operators. Part II. Spectral Theory. Interscience, 1967.

[4] Erdös, L., Magnetic Lieb-Thirring inequalities. Comm. Math. Phys. 170 (1995), 629-668.

[5] Erdös, L., Solovej, J. P., Semiclassical eigenvalue estimates for the Pauli operator with strong non-homogeneous magnetic fields. I. Non-asymptotic Lieb-Thirring type estimates. To appear in Duke Math. J.

[6] Erdös, L., Solovej, J. P., Semiclassical eigenvalue estimates for the Pauli operator with strong non-homogeneous magnetic fields. II. Leading order asymptotic estimates. Comm. Math. Phys. 188 (1997), 599-656.

[7] Fefferman, C., On electrons and nuclei in a magnetic field. Advances in Math. 124 (1996), 100-153.

[8] Fefferman, C., Fröhlich, J., Graf, G. M., Stability of ultraviolet-cutoff quantum electrodynamics with non-relativistic matter. Comm. Math. Phys. 190 (1997), 309-330.

[9] Lieb, E. H., Loss, M., Solovej, J. P., Stability of matter in magnetic fields. Phys. Rev. Lett. 75 (1995), 985-989.

[10] Lieb, E. H., Solovej, J. P., Yngvason, J., Asymptotics of heavy atoms in high magnetic fields: II. Semiclassical regions. Comm. Math. Phys. 161 (1994), 77-124.

[11] Lieb, E. H., Thirring, W., Bound for the kinetic energy of fermions which proves the stability of matter. Phys. Rev. Lett. 35 (1975), 687-689.

[12] Lieb, E. H., Yau, H. T., The stability and instability of relativistic matter. Comm. Math. Phys. 118 (1998), 177-213.

[13] Loss, M., Yau, H. T., Stability of Coulomb systems with magnetic fields: III. Zero energy bound states of the Pauli operator. Comm. Math. Phys. 104 (1986), 283-290.

[14] Reed, M., Simon, B., Methods of Modern Mathematical Physics IV. Analysis of Operators. Academic Press, 1978.

[15] Shen, Z., On the moments of negative eigenvalues for the Pauli operator. To appear in J. Diff. Equations.

[16] Simon, B., Functional integration and quantum physics. Academic Press, 1979.

[17] Sobolev, A. V., On the Lieb-Thirring estimates for the Pauli operator. Duke Math. J. 82 (1996), 607-635.

[18] Sobolev, A. V., Lieb-Thirring inequalities for the Pauli operator in three dimensions. IMA Vol. Math. Appl. 95 Springer, 1997. 
[19] Yafaev, D. R., Mathematical scattering theory: general theory. AMS, 1992.

[20] Ziemer, W. P., Weakly differentiable functions. Graduate Texts in Mathematics. 120 Springer, 1989.

Recibido: 24 de junio de 1.998

Luca Bugliaro, Gian Michele Graf Theoretische Physik

ETH-Hönggerberg

CH-8093 Zürich, SWITZERLAND

lucabu@itp.phys.ethz.ch gmgraf@itp.phys.ethz.ch

and

Charles Fefferman

Department of Mathematics

Princeton University Princeton, NJ 08544, USA cf@math.princeton.edu 